\title{
Equilibrium polymerization of cyclic carbonate oligomers
}

\author{
P. Ballone \\ Institut für Festkörperforschung, Forschungszentrum Jülich, D-52425 Jülich, Germany \\ and Università degli Studi di Messina, Dipartimento di Fisica, Contrada Papardo, I-98166 Messina, Italy \\ R. O. Jones ${ }^{a)}$ \\ Institut für Festkörperforschung, Forschungszentrum Jülich, D-52425 Jülich, Germany
}

(Received 10 April 2001; accepted 11 June 2001)

\begin{abstract}
A model of the polymerization of ring oligomers of bisphenol A polycarbonate (BPA-PC) is used to investigate the influence of dimensionality (2D or $3 \mathrm{D}$ ), density and temperature on the size distribution of the polymer chains. The polymerization step is catalyzed by a single active particle, conserves the number and type of the chemical bonds, and occurs without a significant gain in either potential energy or configurational entropy. Monte Carlo and molecular dynamics simulations show that polymerization of cyclic oligomers occurs readily at high density and is driven by the entropy associated with the distribution of interparticle bonds. Polymerization competes at lower densities with long range diffusion, which favors small molecular species, and is prevented if the system is sufficiently dilute. Polymerization occurs in 2D via a weakly first order transition as a function of density and is characterized by low hysteresis and large fluctuations in the size of polymer chains. Polymerization occurs more readily in $3 \mathrm{D}$ than in $2 \mathrm{D}$, and is favored by increasing temperature, as expected for an entropy-driven process. (C) 2001 American Institute of Physics.
\end{abstract}

[DOI: $10.1063 / 1.1389294]$

\section{INTRODUCTION}

Many polymers are produced on an industrial scale by chemical reactions whose detailed mechanisms are largely unknown. ${ }^{1}$ The analysis of these processes is made difficult by the complexity of the species involved, and the separation of chemical and thermodynamics features poses a continuing challenge to experimental and theoretical investigations. ${ }^{2}$

An example is provided by the ring-opening polymerization (ROP) of bisphenol carbonate cyclic oligomers, which can be used to produce high molecular weight polycarbonate chains. ${ }^{3}$ In this reaction, a suitable active species (for instance, alkali-substituted phenol) is added to a solution of the oligomers (e.g., cyclic BPA tetramers) and catalyzes the opening of the ring (see Fig. 1). The active -OM group $(M=$ alkali metal atom) remains at the end of the chain. Since its chemical properties depend weakly on the length of the attached chain, this basic reaction could be repeated. However, a sequence of such reactions results in oligomer depletion and an enrichment in longer chains, whose backbone bonds are vulnerable to attack by the active heads. Individual chains can then grow, shrink, or even lose their identity due to scission-recombination processes. In the absence of a termination step ${ }^{4}$ the system reaches an equilibrium state characterized by a stationary size distribution and a dynamic association of monomers into continuously changing molecular units that are often referred to as "living polymers."

Our previous density functional (DF) calculations have provided information about the mechanisms and energies involved in the reaction in Fig. 1 and several closely related

\footnotetext{
a) Author to whom correspondence should be addressed. Electronic mail: r.jones@fz-juelich.de
}

cases. ${ }^{5}$ For phenol $(\mathrm{M}=\mathrm{H})$, there is a large potential energy barrier that would produce slow kinetics for any realistic reaction temperature, but the reaction takes place with an energy barrier that is small or zero for $\mathrm{M}=\mathrm{Li}$ and $\mathrm{M}=\mathrm{Na}$. In agreement with previous experimental results, ${ }^{6}$ the calculations show that reactants and products have virtually the same energy. Since polymerization to long chains occurs spontaneously, its origin is puzzling, since entropy should favor a multitude of small molecules rather than a single long chain.

This problem has not been analyzed in detail for BPA-PC polymerization, although model studies of equilibrium polymerization date back many years. ${ }^{7,8}$ Simulations often adopt discrete lattice models, ${ }^{9,10}$ and the most recent studies have focused on the monomer-mediated polymerization of linear chains. These models include the addition and evaporation of single monomers and are appropriate for the polymerization of $\alpha$-methylstyrene, ${ }^{11}$ but they exclude the reaction with longer species ${ }^{12}$ necessary for polycarbonates. $^{13}$

We describe here a continuum model that allows particles to interchange their intramolecular bonds (the covalent bonds in the real polymer) according to rules that mimic the ring-opening reaction of Fig. 1. The reverse and direct reactions are allowed with the same probability, so that an initial population of oligomers evolves towards an equilibrium distribution of chains and rings of different size. The model allows for incorporation and removal of segments of variable length from the chain, and for the coexistence of rings and chains. A repulsive short-range interaction between monomers incorporates excluded-volume effects into the model. For simplicity, we consider only flexible polymers. 

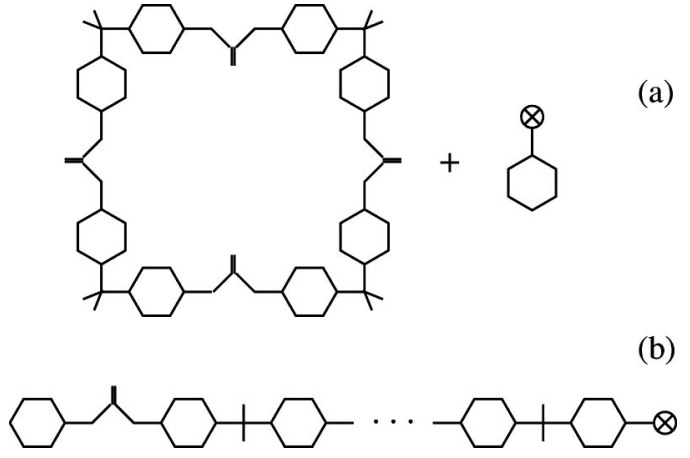

FIG. 1. Schematic view of the first step in the ring opening polymerization of BPA-PC. The active atom, identified by a cross, is an electropositive metal atom.

We focus on very low concentrations of active particles (one in a total population of $10^{4}$ particles), and we analyze in detail the effect of changes in the dimensionality (2D, 3D), density, and temperature $T$ on the degree of polymerization and the equilibrium size distribution. Despite the similarity in the thermodynamic properties of short and long chains, there is a clear tendency towards polymerization that is enhanced by increasing $T$ and particularly by increasing the density. The density dependence of polymerization, which displays a phase transition in 2D that appears to be first order, differs from the results of previous studies for other models of flexible polymers. ${ }^{14}$ The reaction and the associated transition are highly reversible, i.e., the degree of polymerization decreases promptly upon decreasing the system density.

All systems simulated show massive polymerization, with a stronger tendency in 3D than in 2D. The results in 3D are subject to large finite size effects, since the polymers rapidly occupy the entire sample. We discuss the nature of the polymerization transition in our concluding remarks.

\section{THE MODEL AND THE COMPUTATIONAL METHOD}

The BPA-PC structural units are represented in our model by Lennard-Jones (LJ) particles ${ }^{15}$ connected by harmonic springs representing covalent bonds in the polymer backbone. Each particle forms either one or two harmonic bonds, so that the system comprises open chains or rings ${ }^{16}$ without branching. The potential energy of a collection of $(N / 4)$ cyclic tetramers, for example, is

$$
\begin{aligned}
V\left(\mathbf{r}_{1}, \ldots, \mathbf{r}_{\mathbf{N}}\right)= & \epsilon \sum_{i>j}^{N^{\prime}}\left\{\left(\frac{\sigma}{\left|\mathbf{r}_{i}-\mathbf{r}_{j}\right|}\right)^{12}-\left(\frac{\sigma}{\left|\mathbf{r}_{i}-\mathbf{r}_{j}\right|}\right)^{6}\right\} \\
& +\frac{1}{2} k \sum_{\alpha=1}^{(N / 4)}\left\{\left(\mathbf{r}_{4 \alpha-3}-\mathbf{r}_{4 \alpha}\right)^{2}\right. \\
& +\left(\mathbf{r}_{4 \alpha-3}-\mathbf{r}_{4 \alpha-2}\right)^{2}+\left(\mathbf{r}_{4 \alpha-2}-\mathbf{r}_{4 \alpha-1}\right)^{2} \\
& \left.+\left(\mathbf{r}_{4 \alpha-1}-\mathbf{r}_{4 \alpha}\right)^{2}\right\},
\end{aligned}
$$

where the prime on the first sum indicates that the LJ interaction is absent when $i$ and $j$ are connected by the harmonic potential.
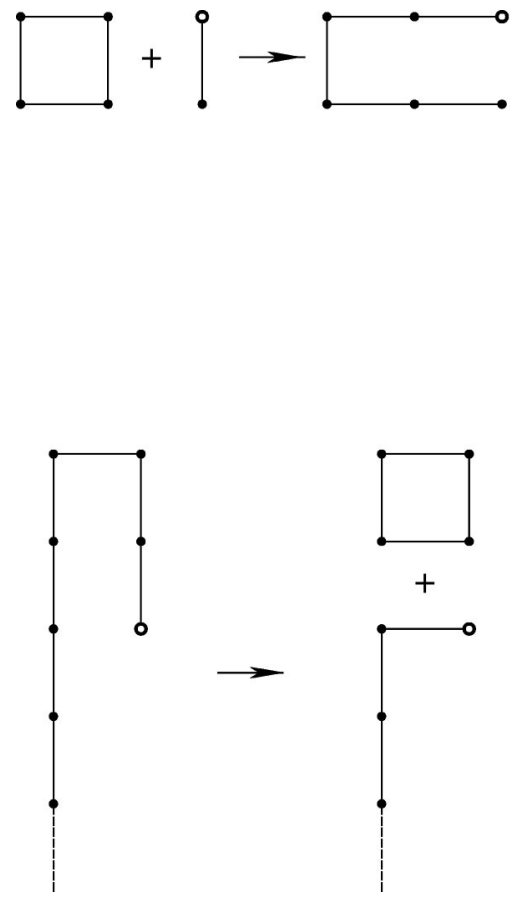

(b)

FIG. 2. Competing bond-interchange processes leading to the growth (a) and to a reduction (b) in length of the active chain.

The units of length and energy are $\sigma$ and $\epsilon$, respectively, and the density is measured in terms of the packing fraction $\eta\left(\eta=\pi \rho \sigma^{2} / 4\right.$ in $2 \mathrm{D}, \eta=\pi \rho \sigma^{3} / 6$ in $\left.3 \mathrm{D}\right)$. The choice of $k$ $(k=3 \epsilon)$ leads to very floppy molecules and allows us to sample efficiently both the intra- and intermolecular degrees of freedom. Angle bending and torsion contributions are neglected. Our simulations, therefore, refer to fully flexible polymers. ${ }^{17}$ We perform simulations for systems with fixed bonding patterns and for which one ("active") particle can interchange its bond with another particle. The active particle can form only one bond, so that it is always located at an end of a chain of at least two particles.

The procedure samples both different bonding configurations and particle positions. At regular intervals we identify the nearest neighbor of the active particle (excluding the particle connected to it by an intramolecular bond), and we link these two particles and two of their covalently bonded neighbors as shown in Fig. 2. The interchange conserves the total number of bonds and is accepted or rejected on the basis of the changes in potential energy and temperature, using the Metropolis algorithm to approach the Boltzmann distribution for the potential energy. The interchange is also rejected if it gives rise to an isolated particle. These rules mean that bond interchange results with equal intrinsic probability in the incorporation of a ring into the active chain segment [Fig. 2(a)] or in the separation of a ring from the active chain [Fig. 2(b)]. The identity of the active and inactive tails of the chain are interchanged with a frequency much lower than the bond-interchange attempts, which enhances the sampling of the system configurations without affecting the monofunctional nature of the active chain. The system is thus treated as a collection of $N$ equivalent particles and $(N-1)$ bonds in thermal equilibrium. 
The potential energy of the system is sampled by a (constant volume) Monte Carlo (MC) method, in which we attempt to translate either single particles or whole molecules with relative probability and steps chosen to achieve an acceptance ratio of $\sim 0.5$ for both types of moves. The interchange of intermolecular bonds is attempted every $N$ single particle steps, with $N$ of the order of the number of particles in the system. The thermodynamic properties of chains of fixed length have been determined in 2D (3D) using systems of 10000 particles in a periodically repeated square (cubic) simulation box.

All simulations incorporating bond interchange were carried out for 10002 particles, starting from 2500 tetramers and a single dimer carrying the active particle, and equilibration was performed initially at the selected temperature and density. The average energy and the structural properties such as the pair distribution function were checked during this stage. Equilibration during polymerization was monitored by counting the tetramers. The initial excess of tetramers is reduced progressively, and their number fluctuates without drift at equilibrium with a concentration that is between those of trimers and pentamers.

The simplicity of the model allows us to perform very long simulations, with millions of steps per particle even for systems of this size. ${ }^{18}$ The above criteria (energy, molecular mass distribution, pair distribution function) show that all results reported below refer to systems at equilibrium. The convergence with respect to the system size is much less certain, especially in 3D. The longest polymer chain exceeds $80 \%$ of the total number of particles in the highest density cases, and these chains would probably grow even longer in larger samples. ${ }^{19}$

Dynamical properties, such as the diffusion or viscosity coefficients, have been computed by microcanonical molecular dynamics (MD) runs for systems of fixed bonding configuration, starting from atomic positions equilibrated by MC and random velocities selected from a Gaussian distribution. The computation of the diffusion coefficient is discussed in more detail in the Appendix. The viscosity coefficient has been computed by integrating the time correlation function of the shear stress, i.e., using the Green-Kubo relation, as discussed in Ref. 20. Together with $\sigma$ and $\epsilon$, the unit mass (the mass of the monomer $m$ ) determines the unit of time $\tau$ $=\sigma(m / \epsilon)^{1 / 2}$. The equations of motion have been integrated by the velocity Verlet algorithm with a time step of 0.5 $\times 10^{-2} \tau$. We refer to the mass of the polymer $M$ or to the mass distribution. Since $m$ is the unit of mass, $M$ is numerically equal to the number of monomers in the polymer.

\section{RESULTS}

\section{A. Monodispersed ring systems of fixed length}

We first investigate the structure and thermodynamic properties of monodispersed systems comprising rings of fixed length. An ideal model system for present purposes would favor neither a specific size nor a specific size range, but it is difficult in practice to tune the potential energy to ensure that the free energy of short and long rings (or chains) is the same over an extended range of $T$ and $\eta$. To quantify

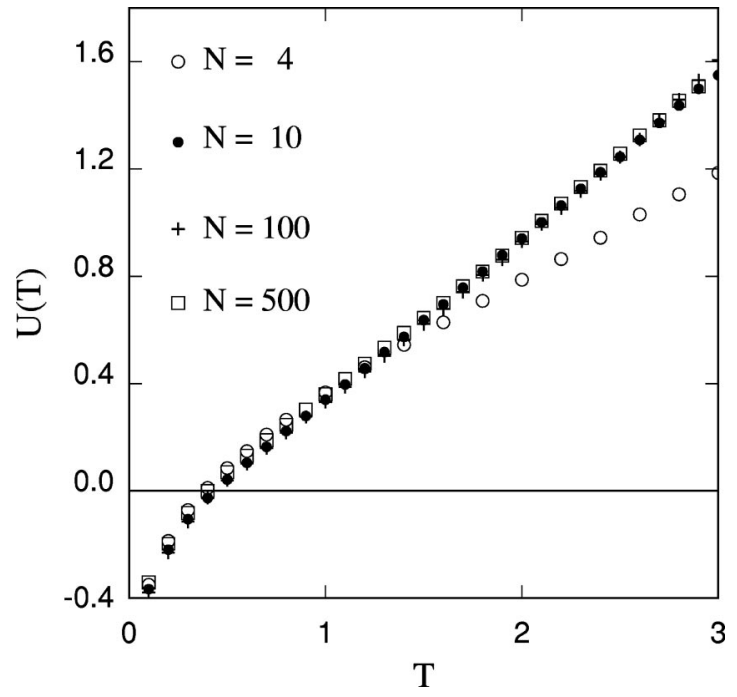

FIG. 3. Average potential energy as a function of temperature for rings of size $N=4,10,100,500$ at $\eta=0.4$.

the size bias in our model, we have performed extensive simulations for rings with $M=4,10,100$, and 500 at $\eta=0.1$ and $\eta=0.4$. The trends are similar for these two densities, and Fig. 3 summarizes the results for the higher packing.

At low $T$ the average potential energy per particle $U(T)$ is relatively insensitive to changes in size. ${ }^{21}$ The dependence is stronger at higher temperatures, but only the smallest size $(M=4)$ deviates significantly from the general trend for large rings, which is already established for $M=10$. The free energy per particle can be obtained from $U(T)$ by thermodynamic integration over the inverse temperature, and the results confirm that the model has almost no size bias, with the possible exception that very short rings appear to be slightly less favored. ${ }^{22}$ The $M$ dependence of the structure supports the thermodynamic evidence, since the radial distribution function varies little beyond the smallest sizes. The diffusion coefficient and the viscosity (see Table I for $\eta=0.4$; similar results are obtained for $\eta=0.1$ ) show that dynamical properties converge to the "long-polymer" limit slower than thermodynamic and structural properties (see Appendix).

\section{B. Simulations involving bond interchanges}

The rules described in Sec. II allow the presence of as many linear segments as active particles, as well as a rings of different sizes. The active chain can grow if it interchanges a

TABLE I. Comparison of diffusion $(D)$ and viscosity $(s)$ coefficients for two-dimensional systems made by $M=4$ oligomers, and systems under equilibrium polymerization conditions. The units for $D$ and $s$ are defined by our choice, $\epsilon=\sigma=\mathrm{m}=1.0$

\begin{tabular}{ccclll}
\hline \hline & \multicolumn{2}{c}{$M=4$ rings } & & \multicolumn{2}{c}{ Equilibrium } \\
\cline { 2 - 3 } \cline { 5 - 6 }$\eta$ & $D$ & $s$ & & \multicolumn{1}{c}{$D$} & \multicolumn{1}{c}{$s$} \\
\hline 0.1 & $3.6 \pm 0.2$ & $0.21 \pm 0.04$ & & $3.4 \pm 0.2$ & $0.24 \pm 0.04$ \\
0.2 & $1.76 \pm 0.1$ & $0.31 \pm 0.06$ & & $0.64 \pm 0.15$ & $0.95 \pm 0.1$ \\
0.3 & $1.28 \pm 0.08$ & $0.52 \pm 0.08$ & & $0.12 \pm 0.01$ & $1.2 \pm 0.2$ \\
0.4 & $0.85 \pm 0.05$ & $0.66 \pm 0.20$ & & $0.05 \pm 0.01$ & $2.4 \pm 0.4$ \\
0.5 & $0.63 \pm 0.05$ & $1.10 \pm 0.20$ & & $0.08 \pm 0.03$ & $4.3 \pm 0.4$ \\
\hline \hline
\end{tabular}


(a) L sites L(L-2) configurations

$\prod_{-1} \prod^{-}$

(b) L sites $\mathrm{L}$ configurations

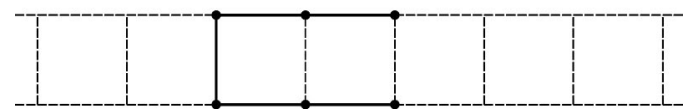

FIG. 4. Number of available configurations for the molecules in the initial and final states corresponding to Fig. 1 on a one-dimensional lattice.

bond with a ring, or become smaller by losing a ring when the active head interacts with a particle of its own chain. Since all covalent bonds are equivalent, and the number of bonds is conserved, the reaction does not change the potential energy and can be driven by entropy alone.

One possible reason for polymerization can be identified using a simple example. If the tetramers and the initiating dimer move on a one-dimensional lattice comprising $L$ sites [Fig. 4(a)] and the particles cannot occupy the same lattice point, the number of configurations available is $L(L-2)$. The elongated chain, on the other hand, has only $L$ available configurations [Fig. 4(b)], and entropy will favor (a) over (b) if the system can assume either configuration without intermixing. However, a reacting system that can transform reversibly from (a) to (b) has the sum of the available configurations, $L(L-2)+L$. If the transformation is allowed, the system will display a non vanishing equilibrium concentration of the elongated chain proportional to $L /[L(L-2)$ $+L]=1 /(L-1)$. Moreover, this concentration will be enhanced if the entropy advantage of the separated units is reduced by high packing (a low $L$ value in the onedimensional example) or by higher dimensionality, which increase rapidly the number of different ways of linking neighboring particles. These considerations provide a basis for understanding our results.

\section{Two-dimensional simulations}

The results of the 2D simulations are much less subject to finite size effects than 3D calculations on systems of the same size. Simulations were performed for $\eta=0.1,0.125$, $0.15,0.2,0.3$, and 0.5 , and for $0.6 \leqslant T \leqslant 4$, for which the particles retain a sizable mobility before polymerization. Most systems polymerize, and an example is shown in Fig. 5 for $\eta=0.4, T=3$ : The initial liquidlike assembly of tetramers (a) evolves into a long chain spanning most of the system, with residual rings of small and medium size (b). At equilibrium, we observe large fluctuations in the length of the open chain, and a sizable mobility of the active head induced by bond interchange. The effect of packing is clear in Fig. 6, which compares the results for $\eta=0.1$ and $\eta=0.2$, at $T=3$. Despite a very long simulation, the open chain does not grow in the dilute system, and the progressive degradation of the tetramers into cyclic trimers is apparent. For $\eta=0.2$, on the other hand, a long chain forms quickly and persists during

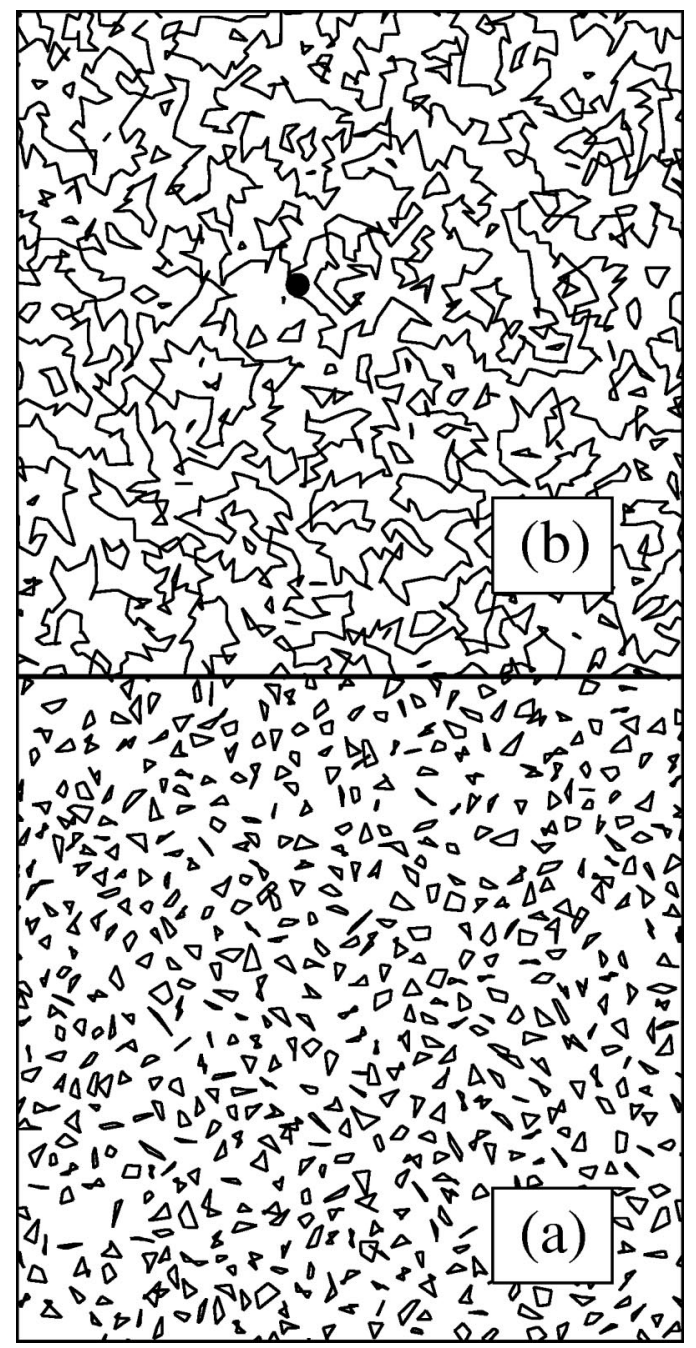

FIG. 5. Snapshots of (a) the initial tetramer fluid, and (b) a configuration at equilibrium under the bond-interchange mechanism for a $2 \mathrm{D}$ system at $\eta=0.4$ and $T=3$. The black dot identifies the active particle. The figure covers only part of the simulated system.

the remainder of a very long simulation, occasionally shedding large rings and growing again by incorporating other rings.

We can provide more detail by computing the average length at equilibrium of rings and the active chain as a function of $\eta$ and $T$. The average length $\left\langle L_{l}\right\rangle$ of the chain rises steadily with increasing density, with an abrupt increase for $0.1 \leqslant \eta \leqslant 0.15$, and a tendency to saturate for $\eta \geqslant 0.4$ (see Fig. 7). The low density anomaly is due to the sudden increase of $\left\langle L_{l}\right\rangle$ from a few monomers at $\eta=0.1$ to a value larger than 2000 at $\eta=0.15$. These indications that the transition is first order are supported by the fact that fluctuations in either $\left\langle L_{l}\right\rangle$ or the potential energy (relatively large for $\left\langle L_{l}\right\rangle$ and fairly small for $U$ ) do not peak close to the transition. The saturation at high density could, however, be due to finite-size effects, since the average length of the open chain exceeds $40 \%$ of the total number of particles under those conditions. The average length $\left\langle L_{r}\right\rangle$ of the rings is two orders of magnitude less than $\left\langle L_{l}\right\rangle$, and it increases monotonically with density (Fig. 7). It is quite regular in the $0.1 \leqslant \eta \leqslant 0.2$ interval, where $\left\langle L_{l}\right\rangle$ displays a jump. 


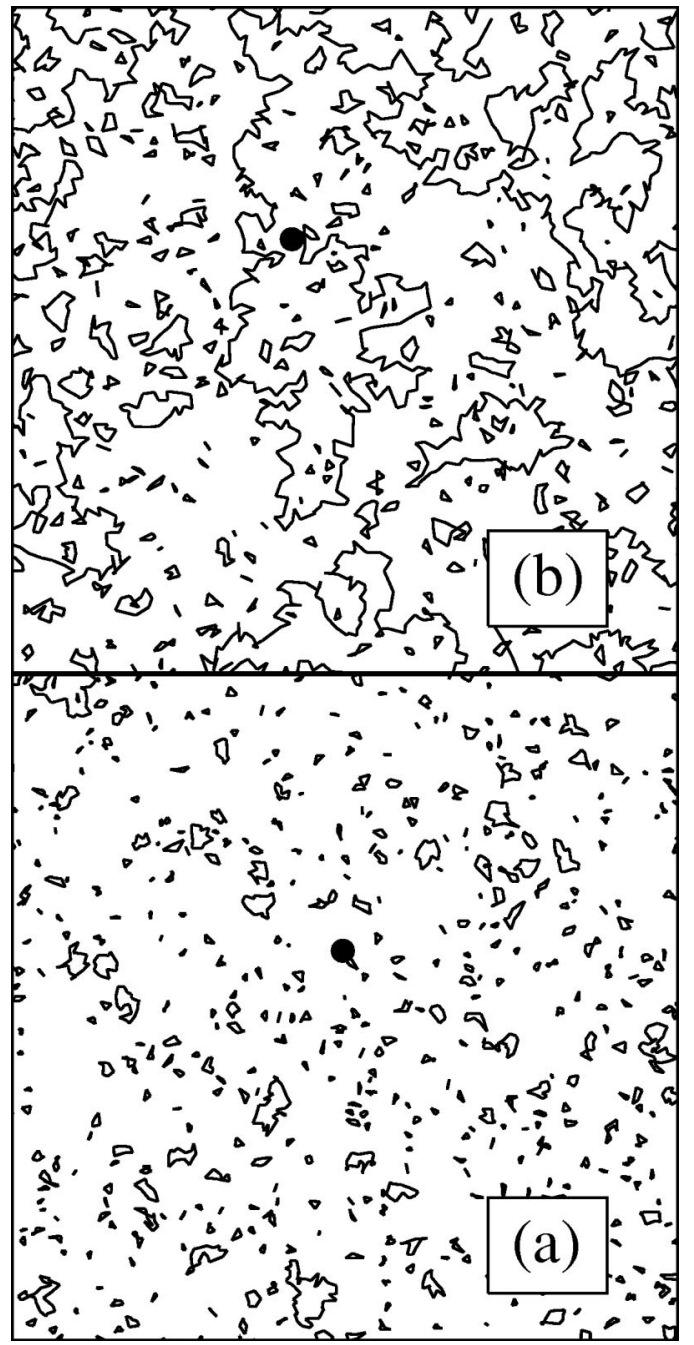

FIG. 6. Snapshots of two systems equilibrated at $T=3$ : (a) $\eta=0.1$, (b) $\eta=0.2$. The black circles identify the active particle. The figure covers only part of the simulated system.

Temperature affects $\left\langle L_{r}\right\rangle$ and $\left\langle L_{l}\right\rangle$ somewhat less strongly than density (see Fig. 8). In the accessible range $(T \geqslant 0.6)$, there is a monotonic increase of $\left\langle L_{l}\right\rangle$ with increasing $T$, a more pronounced slope for $0.6 \leqslant T \leqslant 1$, and a tendency to saturate for $T \geqslant 3$. We cannot identify unambiguously a polymerization line as a function of $T$ at constant density, although we note the sudden change of slope for $\left\langle L_{l}\right\rangle$ below $T=1$. Exploration of still lower temperatures is hampered by the low mobility of the active head, resulting in equilibration times beyond our present capabilities. The presence of an anomaly close to $T=0.6$, however, is suggested also by the nonmonotonic behavior of $\left\langle L_{r}\right\rangle$ around that temperature: the size reduction of the long active chain occurs by the production of several medium size rings that persist in the system and enhance $\left\langle L_{r}\right\rangle$.

We have computed the mass-fraction distribution, defined as the percentage of monomers belonging to a ring $\left(P_{r}(M)\right)$ or a chain $\left(P_{l}(M)\right)$ of mass $M,{ }^{23}$ and the results are shown for well-equilibrated samples in Figs. 9 and 10. Statistical error bars are obviously more significant for individual points in these two curves than for their averages defining $\left\langle L_{r}\right\rangle$ and $\left\langle L_{l}\right\rangle$, and we discuss only the major features

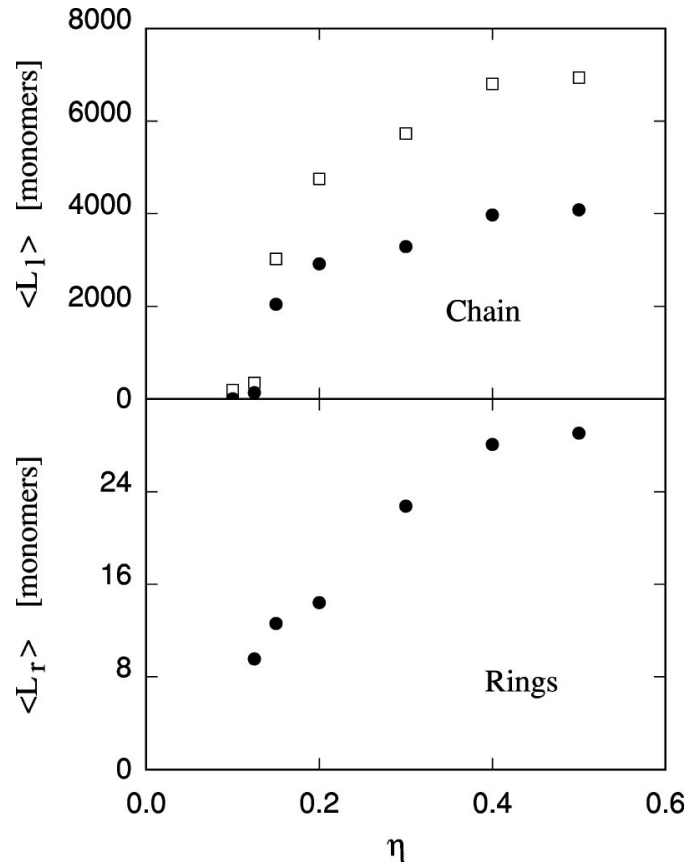

FIG. 7. Solid dots: average length of the chain $\left\langle L_{l}\right\rangle$ and of the rings $\left\langle L_{r}\right\rangle$ as a function of packing fraction $\eta$ at $T=3$. Error bars are of the order of 200 monomers for the chain, and 2 monomers for the rings. Empty squares: maximum length of the active chain during our simulations.

shown by $P_{r}(M)$ and $P_{l}(M)$. The ring distribution shows a high and narrow peak at low $M$, and a nearly constant background extending up to large sizes. The chain distribution has a broad peak for large sizes, and a definite shift towards even larger $M$ with increasing density and $T$ is reflected in $\left\langle L_{r}\right\rangle$ and $\left\langle L_{l}\right\rangle$. The width of the mass distributions [measured by the square root of the second moment of $P_{r}(M)$ and

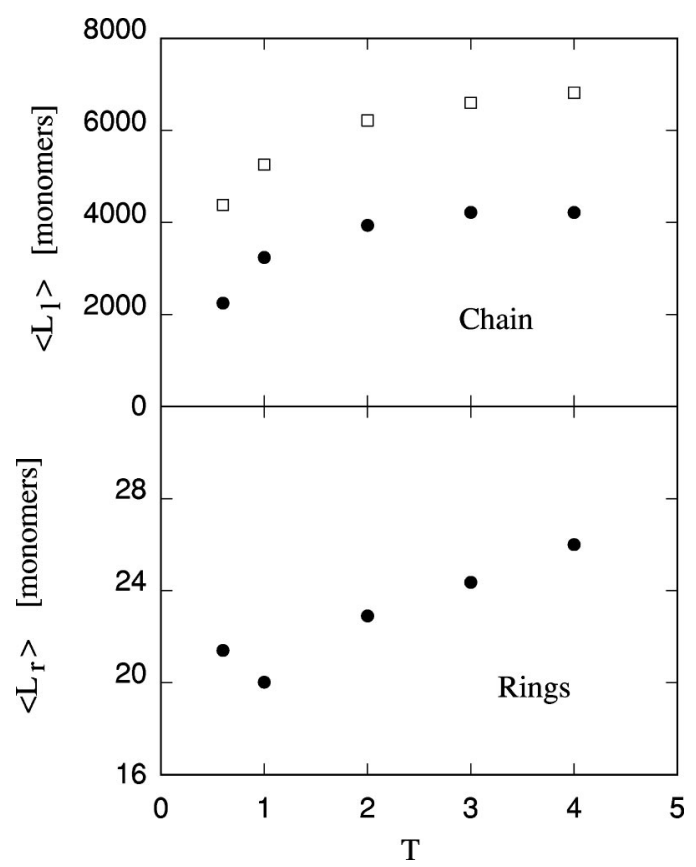

FIG. 8. Solid dots: average lengths of the chain and rings as a function of $T$ at packing fraction $\eta=0.4$. Error bars are similar to those of Fig. 4. Empty squares: maximum length of the active chain during our simulations. 


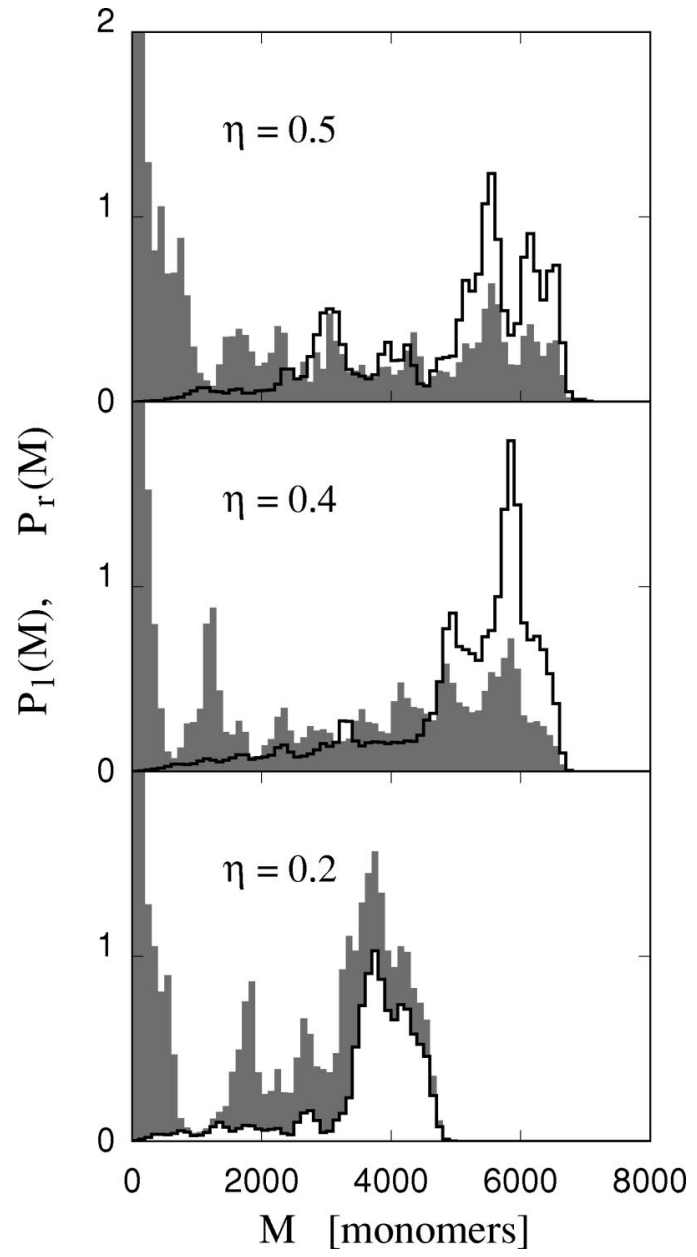

FIG. 9. Mass-fraction distributions for rings $\left[P_{r}(M)\right.$, shaded area $]$ and chains $\left[P_{l}(M)\right]$ as a function of density at $T=3$.

$\left.P_{l}(M)\right]$ is a nonmonotonic function of $\eta$, being slightly wider at intermediate densities than at either low or high packing.

$P_{r}(M)$ and $P_{l}(M)$ broaden as $T$ increases, as shown in Fig. 10. Both distributions span a wide size range in all cases, confirming that the size of rings and open chains fluctuates significantly at equilibrium for all systems that polymerize. Such wide distributions probably arise because the length of the active chain can increase or decrease by large amounts with a single bond interchange. Systems that polymerize by simple addition of monomers display narrower size distributions. The link between broad size distributions and polymerization is also confirmed by the fact that the size ranges of rings and chains is always nearly the same, because of their easy interconversion. Moreover, the two size distributions approach each other at low density and temperature, when the stability of the polymeric state is marginal, and the interconversion between active chain and rings is apparently more effective.

The widths of the mass fraction distribution $P_{r}(M)$ and $P_{l}(M)$ increase monotonically during polymerization. They start from a $\delta$-like distribution at $M=4$, are nearly proportional to the average length of rings and chains during the initial equilibration stages, and increase more rapidly later. Industrial applications exploit similar properties of real

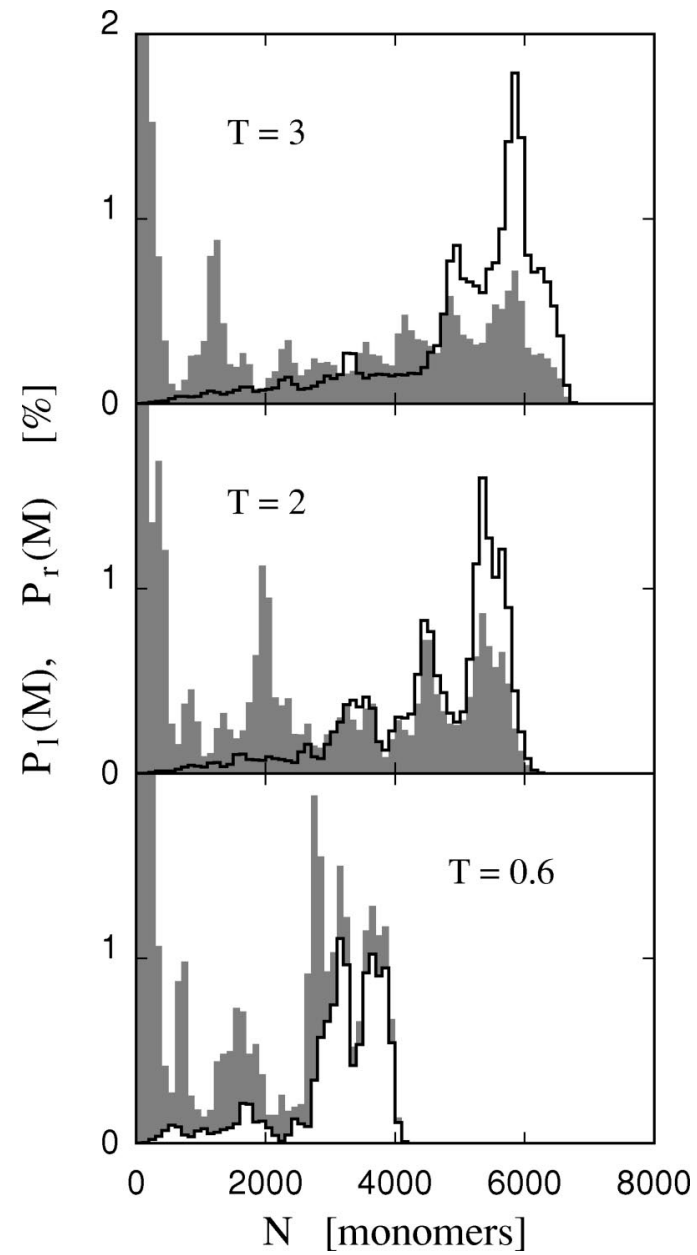

FIG. 10. Mass-fraction distributions for rings $\left[P_{r}(M)\right.$, shaded area $]$ and chains $\left[P_{l}(M)\right]$ as a function of $T$ at $\eta=0.4$.

chemical species in order to stop the polymerization process at the point where good size selectivity accompanies sufficiently long polymer chains. This stage is reached for most materials well before equilibration is achieved.

The size distributions of the rings and of the chain [obtained from $P_{r}(M)$ by dividing by $M$ and normalizing] cannot be approximated by the Flory-Schulz Law often associated with living polymers. The distribution of the open chain is centered on large values of $M$ and differs qualitatively from the nearly exponential decay with increasing size predicted for other polymerization mechanisms and models, including the results of recent nonmean-field theories. ${ }^{24}$ This difference might be related to the different order of the transition here (first order) and in previous simulations (continuous); the fact that the mass-fraction $P_{l}(M)$ is peaked at large sizes for every system that polymerizes is a further indication that the transition is discontinuous.

The degree of polymerization also shows subtle correlations with structural properties, such as the radius of gyration $R_{g}$. This is defined by

$$
R_{g}^{2}=\frac{1}{2 N^{2}} \sum_{i \neq j}^{N}\left\langle\left(\mathbf{x}_{i}-\mathbf{x}_{j}\right)^{2}\right\rangle
$$

and is directly related to the low-q behavior of the structure 


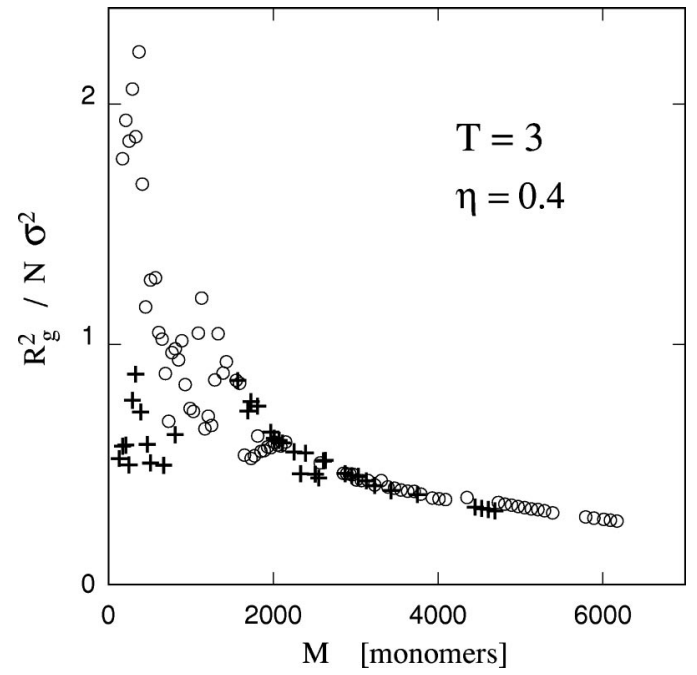

FIG. 11. Square of the radius of gyration per particle $R_{g}^{2} / M$ of rings (crosses) and chains (circles) as a function of length $M$.

factor. $R_{g}^{2}$ increases substantially during polymerization, leading to a large increase in the low-q scattered intensity. For all samples that polymerize in the present study, chains (either open or cyclic) tend to be coiled, i.e., $R_{g}^{2} / N$ is close to unity. It is not surprising that open chains are more extended than rings, especially for the smallest sizes (see Fig. 11). We have also found that short open chains are significantly more extended than long ones, probably because of excluded volume effects. This property probably plays a role in determining the equilibrium length distribution; the active chain reduces its length when the active head interacts with a particle belonging to the chain itself, and this event is less likely in extended polymers. The elongated structure of short chains then provides in-built protection against further length reduction. The structure of open chains is often characterized also by the probability distribution for the end-to-end distance, and we have found that the picture of the polymer size provided by this distribution is similar to the one given by $R_{g}^{2}$.

In practical applications it is important to know the fraction of the reacting system comprising polymers (rings or chains) with a molecular mass exceeding a given threshold. In Fig. 12 we show for our model,

$$
Q(M)=100-\int_{0}^{M} P\left(M^{\prime}\right) d M^{\prime},
$$

for five densities at $T=3$. With the exception of $\eta=0.1$, all systems contain a large fraction of long polymers. The fraction of the system consisting of very short rings $(M<4)$ (Fig. 13) provides an estimate of the portion of original tetramers that are degraded to even smaller species. These very short rings can be viewed as a failure of the polymerization process and always represent a sizable fraction of the total mass. Their number can be reduced by increasing the packing.

Despite their large size difference, rings and chains represent an almost equal fraction of the total mass for all but one of the polymerized samples, since the shorter length of the rings is compensated by their larger number. The only exception to this rule was found in the simulation at $T$

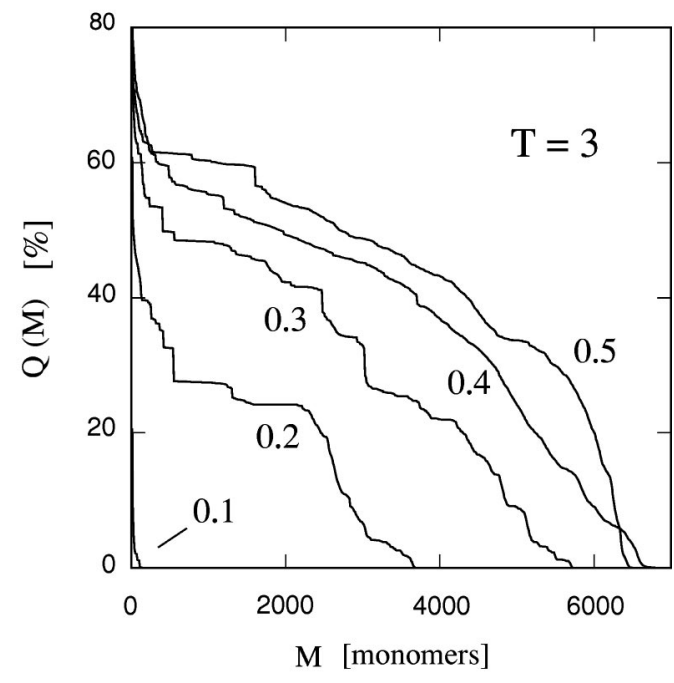

FIG. 12. Fraction $Q(M)$ (in \%) of the system mass represented by polymers (either rings or open chains) of size larger than $M$ (see text). 2D systems at the packing fractions $\eta$ indicated.

$=0.6, \eta=0.4$, for which rings are a factor of 2 greater (in mass) than the open chain. Further computations are needed to decide whether this anomaly is the result of an accidental fluctuation or a feature related to the low temperature of the simulation. An anomaly might indicate an incipient transition to the unpolymerized state at lower $T$, since the abundance of rings implies that the active head intersects the open chain on which it resides more often than usual in polymeric samples.

Insight into the polymerization process can also be gained by analyzing transient features that arise on activating the bond exchange mechanism or by computing equilibrium dynamical coefficients. The most obvious such effect is the progressive increase of the average potential energy, which follows an approximately exponential relaxation law (Fig. 14). The energy rise is expected, since-for the same temperatures-long chains have a higher potential energy that the original tetramers. More surprising are the size of this energy increase and the monotonic relaxation. The first shows that entropy rises quickly and substantially during polymerization, since the combination $F=U-T S$ must decrease during the relaxation towards equilibrium. The

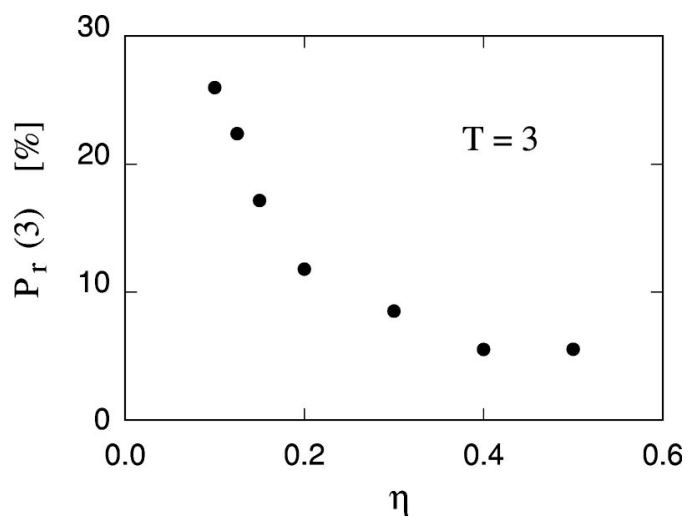

FIG. 13. Fraction of the total mass represented by cyclic trimers as a function of packing fraction $\eta$ for 2D systems. 


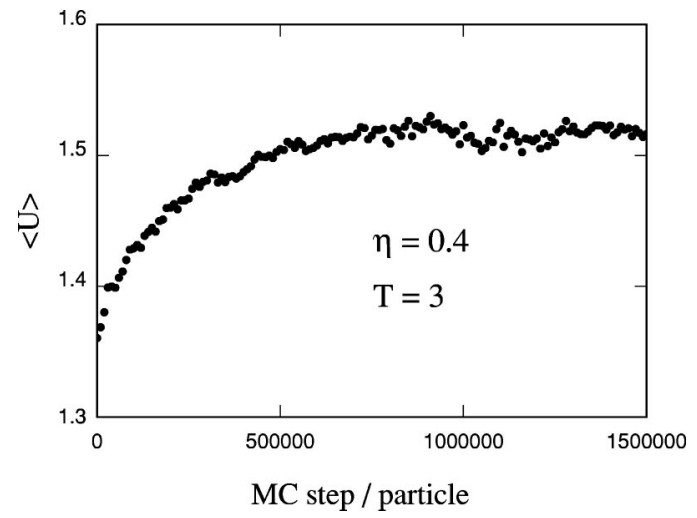

FIG. 14. Potential energy (averaged over $10^{4} \mathrm{MC}$ steps per particle) during equilibration for a $2 \mathrm{D}$ system at $T=3$ and $\eta=0.4$. The simulation starts from a homogeneous liquid comprising cyclic tetramers and evolves towards a polymerized state.

smooth and monotonic relaxation shows that polymerization occurs without crossing significant barriers, but proceeds downhill in the free energy landscape.

The size-dependence of the dynamical properties could provide a sensitive probe of the degree of polymerization. Examples are the diffusion coefficient and the viscosity coefficient, which vary significantly and monotonically during polymerization (Fig. 15). The comparison for the original oligomers $(M=4)$ and the equilibrated polymerization system shows that diffusion is more sensitive to the degree of polymerization (Table I). Large connected chains hamper or even prevent the long range mobility of all other molecules

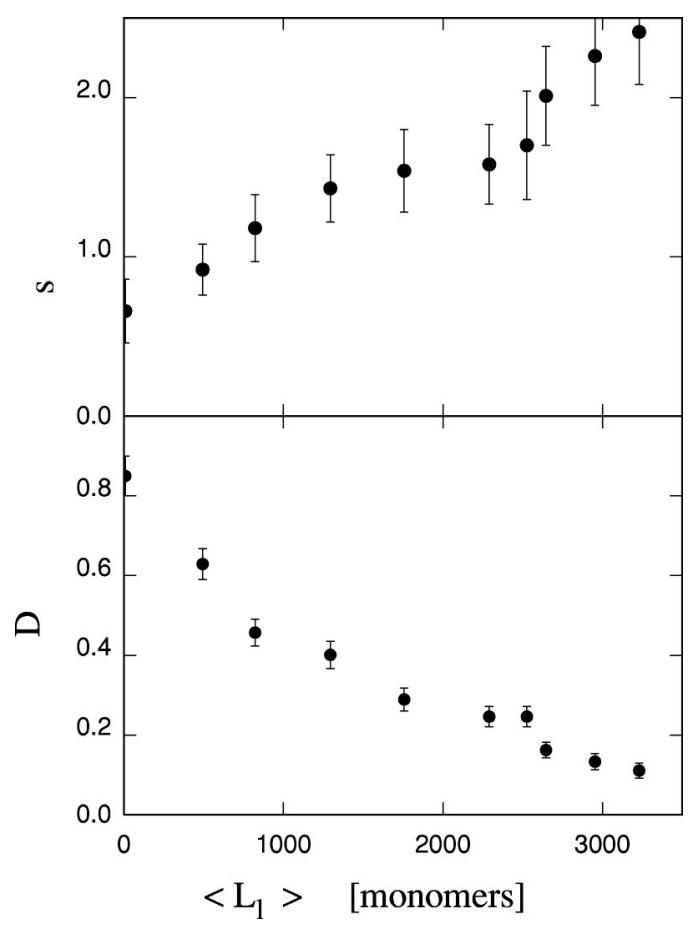

FIG. 15. Diffusion coefficient $D$ and viscosity coefficient $s$ during polymerization at $T=3$ and $\eta=0.4$. Each point has been computed by MD at fixed bonding pattern starting from configurations selected at regular intervals during the MC runs. Each configuration is characterized by the length of the active chain, given by the abscissa. The units of $D$ and $s$ are defined by our units, $\epsilon=\sigma=m=1$.

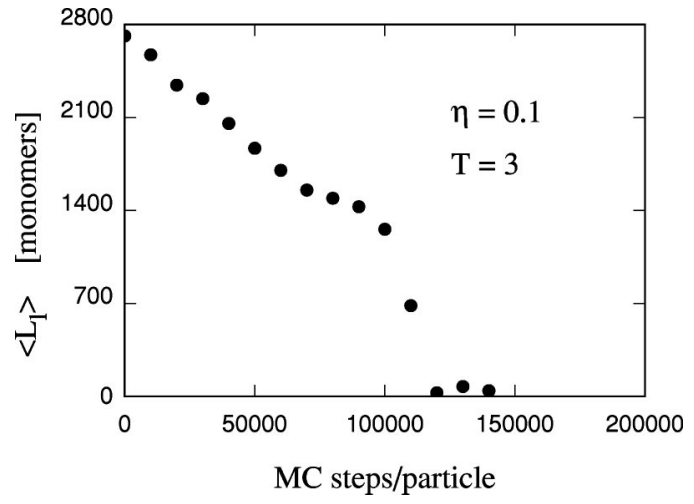

FIG. 16. Evolution of the average length $\left\langle L_{l}\right\rangle$ during a MC run upon expanding a $2 \mathrm{D}$ system from $\eta=0.15$ to $\eta=0.1$ at $T=3$.

in the system, including the surviving oligomers. On the other hand, viscosity is affected by short and medium range interactions, and depends more on packing than on polymerization. Although a precise comparison is prevented by the simplified model we use and the difference in the polymerization mechanism, there are qualitative similarities between our results and measurements of the diffusion ${ }^{25}$ and viscosity $^{26}$ coefficients in $\alpha$-methylstyrene during polymerization.

The large fluctuations in the polymer size distribution suggest that polymerization in our model system should be reversible. This has been demonstrated by starting a MC run from an equilibrium configuration at $\eta=0.15, T=3$ (i.e., near to the polymerization line and on the polymer side), and suddenly decreasing its density to $\eta=0.1$, thereby crossing the polymerization line. ${ }^{27}$ The average length of the active chain, shown in Fig. 16 as a function of the length of MC run, decreases quickly, first following a nearly exponential law, and then collapsing almost discontinuously.

\section{Three-dimensional simulations}

Simulations in 3D have been performed at $\eta=0.03,0.05$, $0.1,0.2,0.3,0.5$ at $T=3$, and at $T=1,2,3$, and 4 for $\eta=0.4$. The much larger samples required to eliminate finite size effects would lead to simulations at least one or two orders of magnitude longer than the (already time-consuming) computations performed here. Nevertheless, the results provide a picture of qualitative behaviors and trends on going from two to three dimensions. First of all, at equal $T$ and $\eta$ the model displays a more pronounced tendency towards the polymeric state in 3D than in 2D. The average length $\left\langle L_{l}\right\rangle$ of the active chain, plotted as a function of the density in Fig. 17, is always greater in $3 \mathrm{D}$ than in $2 \mathrm{D}$, although the existence of a polymerization line at low density cannot be identified unambiguously. ${ }^{28}$ The stronger tendency to polymerization is also apparent in the rise of the potential energy during equilibration, which is at least a factor of 2 larger in 3D than in 2D. The mass-fraction distributions of rings and the open chain are wider and smoother in 3D, although the overall shape of these functions is similar. For the same values of $T$ and $\eta$, diffusion is easier in 3D and relaxation towards equilibrium faster. 


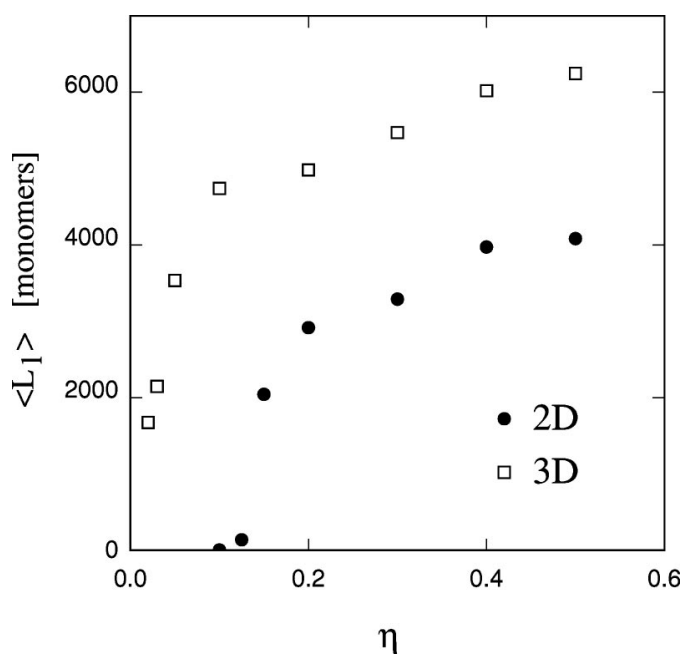

FIG. 17. Comparison of the average length of the active chain as a function of packing fraction in $2 \mathrm{D}$ and in $3 \mathrm{D}$ at $T=3$.

The simulations in 3D and 2D show similarities as well as differences. The average polymer length increases with increasing density and, to a lesser degree, with increasing $T$. The saturation in the degree of polymerization evident at the upper limit of the density and temperature ranges could be due to finite size effects. The average size of rings is again roughly two orders of magnitude smaller than the average length of the open chain, but in 3D the total mass of the rings is somewhat less than in the active chain. The computation of the radius of gyration shows that the overall picture discussed above for the 2D case is also valid in 3D. Changes in dynamical properties due to polymerization are qualitatively similar in 2D and 3D, but the quantitative effects are clearly enhanced in 3D because of the stronger tendency towards the polymerized state.

\section{DISCUSSION AND CONCLUDING REMARKS}

Equilibrium and nonequilibrium properties for systems of $N$ Lennard-Jones particles connected by $(N-1)$ harmonic bonds have been investigated by MC and MD simulations. Each particle forms at least one and at most two harmonic bonds, so that our systems comprise a variable number of rings plus one open chain. The model allows the connectivity of the harmonic links to be interchanged by the interaction with one active particle at one of the free ends of the chain. The rules governing the bond interchange model the nucleophilic attack of ring oligomers of BPA-PC; all harmonic bonds are equivalent, their number is conserved during the simulation, and the energy barrier for bond interchange is zero. We have investigated systems of 10002 particles in 2D and in 3D, with packing fractions $\eta$ and temperatures $T$ in the ranges $0.005 \leqslant \eta \leqslant 0.5$ and $0.6 \leqslant T \leqslant 4$.

Computations for systems comprising only rings of length $M$, and excluding the bond interchange mechanism, show that thermodynamic properties (average potential energy and Helmholtz free energy per LJ particle) depend very weakly on the ring length, at least for $M \geqslant 10$. Equilibrium dynamical properties (diffusion coefficient and viscosity) display larger but regular dependences on $M$. Turning on the bond interchange mechanism in a system of cyclic tetramers results in the rapid formation of many medium size ( $M$ $\sim 50$ ) rings and a very long chain (carrying the active head) for most of the thermodynamical conditions we have investigated. In 2D we find a massive polymerization for densities corresponding to $\eta \geqslant 0.125$, and the degree of polymerization increases with increasing density beyond this point. Polymerization induced by density changes appears to be associated with a weakly first-order phase transition, characterized by low hysteresis and large fluctuations in the size of individual polymer chains. Polymerization is also enhanced by increasing temperature, but the effect is much less important than that of increasing density.

The equilibrium probability distribution for the size of rings decreases rapidly with size for small values of $M$, and displays a broad and structureless background up to large sizes. The size distribution for chains has a broad peak at large sizes in all systems that polymerize. Neither distribution functions can be approximated by the nearly exponential decaying functions predicted for models of $\alpha$-methylstyrene. Thermodynamic functions and transport coefficients display regular trends in the transient stage during which the size distribution reaches equilibrium; potential energy and viscosity increase monotonically during polymerization, while the diffusion coefficient is reduced drastically. Threedimensional systems show much greater tendencies to polymerization than two-dimensional systems.

The present results differ from earlier studies of qualitatively different flexible living polymer models mainly in predicting a first order polymerization transition with increasing density. Mean-field studies and beyond for models appropriate to $\alpha$-methylstyrene have generally predicted a second order transition with a lambda anomaly in the specific heat. ${ }^{8}$ The main differences are that the mechanism we have adopted conserves the number of bonds in the system and proceeds via the catalytic activity of a special particle. Moreover, it allows for the coexistence of rings and open chains and assumes that the polymerization step is neutral concerning potential energy, so that differences from the results of previous models are to be expected.

We now discuss the driving force behind the transition. We have noted that the model does not introduce a substantial thermodynamical bias with respect to the polymer size, with the possible exception that very small rings are less favored. Since the potential energy rises upon polymerization, the transition must be due to an entropy contribution related to the bond interchange mechanism. First, the mechanism allows a wide range of polymer sizes to be explored, thus increasing enormously the number of possible configurations (see the discussion of the 1D example of Fig. 4). Moreover, in dense homogeneous systems the number of long, open chains of bonds connecting nearest neighboring particles is exponentially larger than the number of short cyclic paths. The formation of long chains, however, reduces the long range mobility of particles (as shown by the strong decrease in the diffusion coefficient) that is another crucial source of entropy. As a result, polymerization prevails at high density, when long range mobility is already prevented by packing. The importance of the mobility of short chains at 
low density is reflected in the decline of polymerization. The rapid increase in the number of long open paths with the number of neighbors within a bonding distance also explains the greater tendency to polymerization found in three dimensions.

Additional contributions will be provided by dynamical effects, since the local mobility of particles is less constrained in long open chains than in short cyclic ones. Again, the optimization of local mobility (leading to polymerization) prevails at high density, when long range mobility is ineffective. These considerations all suggest a similarity to crystallization, ${ }^{29}$ which occurs even for hard spheres when the potential energy is identically zero and so must be entropy driven. Crystallization can be understood as a similar competition between short and long range constraints, and this parallel supports our identification of polymerization as a first-order transition. It also indicates why density is much more important than temperature.

The (first order) polymerization transition displays very little hysteresis, as shown by the rapid depolymerization of a sample expanded below the transition density. Reversibility, together with a low or vanishing polymerization enthalpy, are properties shared by important biopolymers such as actin or tubulin, which polymerize and depolymerize in living cells under the influence of subtle chemical signals in the presence of suitable catalysts (enzymes) that reduce the potential energy barrier dramatically. ${ }^{30}$ Our model lacks essential ingredients (including rigidity or an environmentdependent interaction) of these systems, but it might shed light on the driving force behind these processes.

Our study suggests interesting directions for future work. Apart from the obvious extension to larger systems and additional active sites, there are questions concerning the existence, location and nature (continuous or discontinuous) of a polymerization line in 3D. There are indications (see Fig. 17) of a polymerization transition qualitatively similar to that observed in $2 \mathrm{D}$, and this is supported by the fact that the mass fraction of rings grows when the density is reduced below $\eta=0.2$. Furthermore, the effects of solvents and impurities, and the interplay with other phase transitions are interesting fields. Our simulations do not reveal clear discontinuities corresponding to liquid-solid transitions, because the system becomes microcrystalline, and disordered already at medium range.

\section{APPENDIX: THE SELF-DIFFUSION COEFFICIENT}

We discuss here the connection of the present results to the self-diffusion coefficient, which is defined for a system of $N$ equivalent particles as

$$
D_{P}=\lim _{t \rightarrow \infty} \frac{\Delta_{P}^{2}(t)}{t}=\frac{1}{t} \sum_{i=1}^{N} \frac{\left\langle\left|r_{i}(t+\tau)-r_{i}(\tau)\right|^{2}\right\rangle}{2 N d},
$$

where $d$ is the system dimensionality and the average is over the initial time $\tau$. If these particles are grouped into $(N / M)$ molecules of size $M,{ }^{31}$ we can define a similar diffusion constant $\left(D_{M}\right)$ for the molecules in terms of the molecular centers-of-mass $\left\{\mathbf{R}_{\mathbf{I}}, I=1, \ldots,(N / M)\right\}$,

$$
D_{M}=\lim _{t \rightarrow \infty} \frac{\Delta_{M}^{2}(t)}{t}=\frac{1}{t} \sum_{I=1}^{(N / M)} \frac{\left\langle\left|R_{I}(t+\tau)-R_{I}(\tau)\right|^{2}\right\rangle}{2(N / M) d} .
$$

The two coefficients must be equal if the bonding configuration cannot change, because the displacement of each particle from the corresponding center of mass is finite, and $\left[\Delta_{P}^{2}(t)-\Delta_{M}^{2}(t)\right] / t$ must vanish for $t \rightarrow \infty$. On the other hand, $\Delta_{P}^{2}$ and $\Delta_{M}^{2}$ can differ if bonding in the system can change.

The time dependence of the positions of the particles has been simulated here by microcanonical MD at fixed bonding among the particles, and a linear approximation to the long time behavior of $\Delta_{P}^{2}(t)$ or $\Delta_{M}^{2}(t)$ should provide an estimate of the diffusion constant for the system under study. The two are equivalent in practice, and the estimate is reliable if both functions approach linearity within the integration time in our simulations $\left(\sim 10^{6}\right.$ time steps, equivalent to 5000 time units). ${ }^{32}$ This is true for small chains $(N \leqslant 20)$ for $T \geqslant 1$ and $\eta \leqslant 0.5$, but not for longer chains, especially at high packing. For longer polymers, we find that $\Delta_{P}^{2}(t)$ is almost linear already at short times, but the slope decreases slowly with increasing simulation time, indicating a diffusion coefficient that is much lower than the one we could estimate directly by Eq. (A1). On the other hand, $\Delta_{M}^{2}(t)$ is already linear at short times, and its slope corresponds well to the long time limit of the slope of $\Delta_{P}^{2}(t)$ (as we have shown for $N=100$ ), so we use $\Delta_{M}^{2}(t)$ to estimate the diffusion constants for systems with $N \geqslant 20$ in Table I.

Over a time span that increases with $M, \Delta_{P}^{2}(t)$ is slightly larger for longer chains than for smaller ones, i.e., over microscopic times, particles in longer chains explore a local environment that is larger than particles in shorter ones. The motion of a monomer in a long chain is less constrained at short times by the slow motion of the center-of-mass, and the entropy advantage is particularly true at high density, for which long time diffusion is practically unimportant even for short chains. The bond interchange mechanism breaks the relation between single particle diffusion and center of mass displacement even at long times, individual particles can hop from one (dynamically defined) molecular aggregate to another, and $D_{P}$ can be larger than $D_{M}$. The difference can be large for highly polymerized samples, for which the time scale over which the molecular constraint affects the single particle displacement becomes much longer than the lifetime of individual bonds. In such cases, there is a gain in entropy because particles in long chains explore a wider portion of the phase space, thus displacing the chemical equilibrium even farther towards larger sizes.

\footnotetext{
${ }^{1}$ Comprehensive Polymer Science, edited by G. Allen and J. C. Benington (Pergamon, Oxford, 1989).

${ }^{2}$ S. C. Greer, in Advances in Chemical Physics, edited by I. Prigogine and S. A. Rice (Wiley, New York, 1996), Vol. 94, p. 261; S. C. Greer, J. Phys. Chem. B 102, 5413 (1998).

${ }^{3}$ D. J. Brunelle, in Ring-Opening Polymerization: Mechanisms, Catalysis, Structure, Utility, edited by D. J. Brunelle (Hanser, München, Germany, 1993), p. 309.

${ }^{4}$ In the industrial synthesis of polycarbonates, the polymerization process is terminated at an early stage, in order to optimize the size distribution of the polymer chains.

${ }^{5}$ P. Ballone, B. Montanari, and R. O. Jones, J. Phys. Chem. A 104, 2793 (2000); P. Ballone and R. O. Jones, ibid. 105, 3008 (2001).
} 
${ }^{6}$ D. J. Brunelle, E. P. Boden, and T. G. Shannon, J. Am. Chem. Soc. 112, 2399 (1990).

${ }^{7}$ Early chemical kinetics models and mean-field solutions are reported in W. B. Brown and M. Szwarc, Trans. Faraday Soc. 54, 416 (1958); P. J. Flory, Principles of Polymer Chemistry (Cornell University Press, Ithaca, 1953).

${ }^{8}$ Theories beyond mean-field have been applied in J. C. Wheeler, S. J. Kennedy, and P. Pfeuty, Phys. Rev. Lett. 45, 1748 (1980), following the renormalization approach introduced by P. G. de Gennes, Phys. Lett. 38A, 339 (1972); J. des Cloizeaux, J. Phys. (Paris) 36, 281 (1975).

${ }^{9}$ (a) A. Milchev and Y. Rouault, J. Phys. II 5, 343 (1995); (b) A. Milchev and D. P. Landau, Phys. Rev. E 52, 6431 (1995); (c) S. Livne, Macromolecules 27, 5318 (1994).

${ }^{10}$ Y. Rouault and A. Milchev, Phys. Rev. E 51, 5905 (1995).

${ }^{11}$ K. M. Zheng and S. C. Greer, Macromolecules 25, 6128 (1992); S. S. Das, A. P. Andrews, and S. C. Greer, J. Chem. Phys. 102, 2951 (1995); S. C. Greer, Comput. Mater. Sci. 4, 334 (1995); S. S. Das, J. Zheung, A. P. Andrews, S. C. Greer, C. M. Guttman, and W. Blair, J. Chem. Phys. 111, 9406 (1999).

${ }^{12}$ A. Milchev, Y. Rouault, and D. P. Landau, Phys. Rev. E 56, 1946 (1997); J. Dudowicz, K. F. Freed, and J. F. Douglas, J. Chem. Phys. 111, 7116 (1999); A. Milchev, J. P. Wittmer, and D. P. Landau, ibid. 112, 1606 (2000).

${ }^{13}$ The sensitivity on the details of the polymerization reaction is emphasized in J. F. Henderson and M. Szwarc, Macromolecules 3, 317 (1968), where it is shown that linear polymers with two active heads behave qualitatively differently from those with one active head.

${ }^{14} \mathrm{~A}$ first order polymerization transition has been predicted for semiflexible species. See Ref. 9(b).

${ }^{15}$ The LJ potential is set equal to zero for $r \geqslant 3 \sigma$. It is replaced by a third order polynomial for $2.8 \sigma \leqslant r \leqslant 3 \sigma$ such that the potential and its first derivative are continuous throughout.

${ }^{16}$ For simplicity, we exclude the possibility of isolated particles.

${ }^{17}$ Nonvanishing bending and torsional force constants could introduce qualitatively different features. See, for example, Ref. 9(b) and M. V. Jaric and K. H. Bennemann, Phys. Rev. A 27, 1228 (1983); M. V. Jaric and K. H. Bennemann, Phys. Lett. 95A, 127 (1983).

${ }^{18}$ Computations have been performed on Compaq XP1000 workstations with a single CPU (EV6 or EV67).

${ }^{19}$ A quantitative assessment of finite size effects that is planned will require very long computations, at least for some benchmark cases.

${ }^{20}$ M. P. Allen and D. J. Tildesley, Computer Simulation of Liquids (Clarendon, Oxford, 1989).
${ }^{21}$ Although the ground state structures obtained by simulated annealing appear to be different for different values of ring size $M, U(T)$ is remarkably insensitive to changes in $M$. This is because all ground state structures can be described as porous microcrystalline materials, with similar local coordination.

${ }^{22}$ In addition to the configurational entropy, there is an ideal contribution that contributes little to the free energy difference at the densities and temperatures of our study.

${ }^{23}$ The mass fraction $P(M)$ defined in Sec. III is different from the size probability distribution $C(M)$ (defined as the concentration of polymers of mass $M$ ) often discussed in computational papers. The two distributions are related by a factor proportional to $M . P(M)$ is more relevant for applications, as it gives a visual impression of the relative mass contribution from each size.

${ }^{24}$ L. Schäfer, Phys. Rev. B 46, 6061 (1992).

${ }^{25}$ J. Ruiz-Garcia and R. Castillo, J. Chem. Phys. 110, 10657 (1999); the diffusion coefficient is directly related to the $g^{2}$ coefficient shown in Fig. 1.

${ }^{26}$ J. Ruiz-Garcia and S. C. Greer, J. Mol. Liq. 71, 209 (1997). Earlier measurements of the viscosity coefficient for $\alpha$-methylstyrene are reported in K. M. Zheng, S. C. Greer, L. C. Corrales, and J. Ruiz-Garcia, J. Chem. Phys. 98, 9873 (1993).

${ }^{27}$ For a study of the response to large deviations from equilibrium, see A. Milchev, Y. Rouault, and D. P. Landau, Phys. Rev. E 56, 1946 (1997), who derived rate equations for monomer-mediated polymerization.

${ }^{28}$ The region of very low density cannot be sampled efficiently by our simulations because the frequency with which the active head collides with other particles is too low to lead to equilibration within an acceptable time.

${ }^{29}$ The relation between polymerization and crystallization has been discussed in early studies of the phase transition in actin, F. Oosawa, S. Asakura, K. Hotta, N. Imai, and T. Ooi, J. Polym. Sci. 37, 323 (1959); F. Oosawa, S. Asakura, and T. Ooi, Prog. Theor. Phys. Suppl. 17, 14 (1961).

${ }^{30} \mathrm{~A}$ recent experimental study of actin polymerization is reported in R. Ivkov, J. G. Forbes, and S. C. Greer, J. Chem. Phys. 108, 5599 (1998).

${ }^{31}$ We restrict ourselves to the monodisperse case, but the generalization to the polydisperse case is straightforward.

${ }^{32} \mathrm{~A}$ variety of methods has been devised to overcome this limitation in MD, and to allow a comparison of diffusion coefficients by MC simulations. 